\title{
An In-Depth Analysis of the Biometric Authentication Classification ${ }^{1}$
}

\section{Ram Khanna}

DOI: $10.37648 /$ ijrst.v10i03.006

Received: 04 ${ }^{\text {th }}$ July, 2020; Accepted: 07 ${ }^{\text {th }}$ August, 2020; Published: $15^{\text {th }}$ September, 2020

\section{ABSTRACT}

Out of the numerous confirmations conspires in this paper, we are attempting to focus on the exhibition and grouping of one of the validation strategies, biometric verification. Even though endeavours of the whole global biometric local area, the estimation of the precision of a biometric framework is far to be totally examined and, ultimately, normalized. The paper presents a basic examination of the analysis of accuracy and execution of a biometric framework.

\section{INTRODUCTION}

\section{A. Arrangement}

If the framework has many clients, it may be smart to make a type of grouping of the example before beginning to contrast it with the simple formats in the information base. That way, the number of vital structures to be tried can be enormously decreased and the preparing time.

The figure shows the traditional finger impression characterization framework that law implementation offices have utilized for quite a long time. At the point when a unique finger impression was imprinted on the card to be placed into a file, a specialist originally inspected it to arrange it. It was much simpler to track down a coordinating with format when another finger impression showed up. Today the characterization is done naturally, and the technique relies upon the sort of biometrics framework utilized.

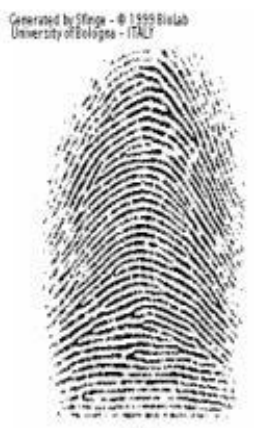

Arch

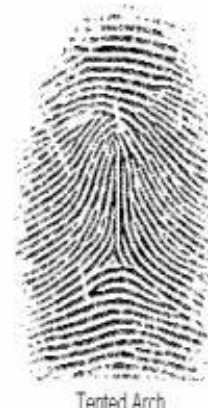

Tented Arch

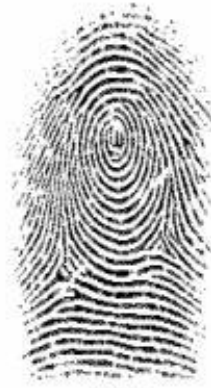

Whot

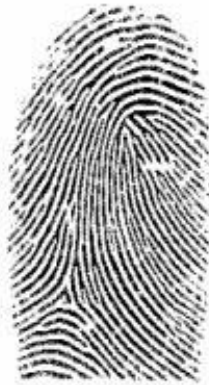

Right Loop



Lett Loog

Fig 1: Biometric classification Example

\footnotetext{
${ }^{1}$ How to cite the article: Khanna R., An In-Depth Analysis of the Biometric Authentication Classification, IJRST, Jul-Sep 2020, Vol 10, Issue 3 , 47-51, DOI: http://doi.org/10.37648/ijrst.v10i03.006
} 


\section{B. Coordinating:}

The coordinating with technique is the piece of the check interaction where the framework attempts to discover a format in its information base that is "adequately" the same as the example given by the client. Because of the simple idea of the client test, the framework will presumably not track down an ideal match in its information base yet rather a rundown of possible matches. If the framework acknowledges the client or not relies upon a type of safety edge set by the framework overseer.

How the coordinating with technique is performed rely much upon what sort of biometrics framework we are discussing. By and large, the framework would attempt to track down some vital components in the client test to coordinate against the layouts.

\section{Exchange Completion and Storage}

If the framework is intended for confirmation or distinguishing proof, the aftereffect of the exchange can be to acknowledge, dismiss or list possible matches. Because of a check framework, it very well may be a smart thought to save a log of endeavoured confirmations for security reasons and measurable reasons. A few frameworks may likewise refresh the format upon a fruitful exchange; this way, the layout quality will continually improve. The framework will want to deal with little normal changes to the biometric. For instance, scars in fingerprints, maturing and so on.

\section{Framework Performance}

Framework execution is an obscure term and what it implies relies much upon what sort of framework it alludes to. When discussing biometrics framework execution, one, for the most part, means the likelihood that the framework will acknowledge approved clients and reject unapproved clients. As referenced before, a biometrics framework normally has some security edge setting that empowers the framework overseer to change the framework to ideal execution.

The False Reject Rate (FRR) and the False Accept Rate (FAR) are frequently referenced while depicting biometrics frameworks. The FRR is, as one would figure from the name, the level of times the framework won't acknowledge an approved client, and the FAR is the level of times that the framework will recognize an unapproved client. The FAR and the FRR are firmly associated. If the framework executive ascents the security edge, the bogus acknowledges will drop. Shockingly, simultaneously the FAR will increment since it additionally will be more earnestly for the live examples of approved clients to coordinate with the greater levels of popularity. The opposite is additionally obvious. If the edge is brought down, the FRR will drop, yet the FAR will rise.

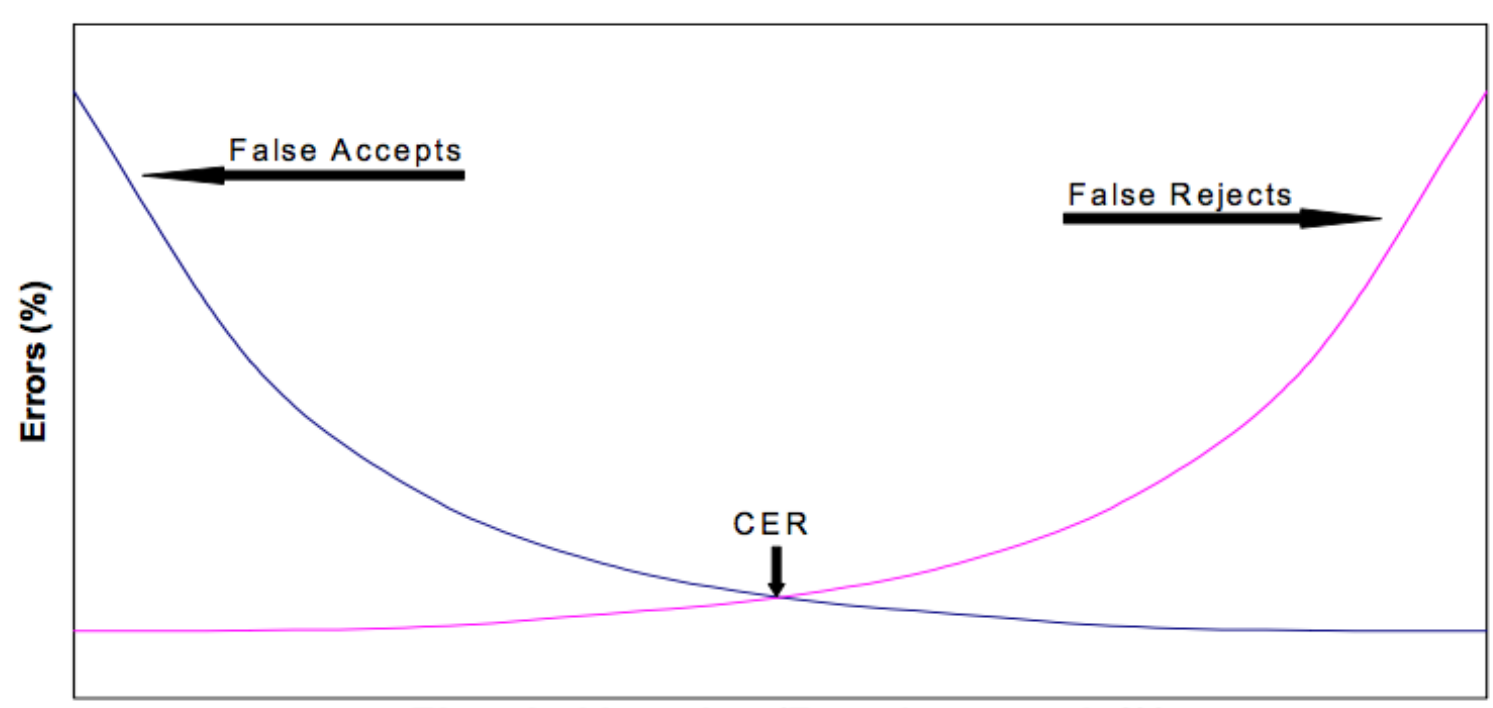

Threshold setting (Template match \%)

The Crossover Error Rate (CER), or as it is in some cases alluded to, the Equal Error Rate (EER), is where the FRR and the FAR bend meet. The figure shows a model of how these terms are connected. When 
attempting to set the security edge to get the ideal execution out of a biometrics framework, it has been shown that the CER point is typically the perfect decision [1]. This isn't generally the situation; it relies upon the kind of safety levels that are required. On the off chance that the framework is expected to confirm the personality of the approved staff at Fort Knox, a couple of bogus oddballs are most likely to incline toward contrasted with the danger of giving unapproved workforce admittance the offices. Then again, if the biometrics framework is utilized in an ATM, the threat of a couple of bogus acknowledges most likely to lean toward contrasted with the disturbance of the clients holding up in line if the framework continues to dismiss approved clients.

Another important term when discussing framework execution, however frequently not referenced by merchants, is Failure To Acquire biometric (FTA). The explanation merchants do not notice this number is that it is typically significantly higher than the FAR and FRR. Say, for instance, that the sellers of a finger impression confirmation framework guarantee their framework has a CER of $0.0001 \%$. That could be valid in principle, however relying upon the examining gadget and the ability of client bunch, the FRR could be $20 \%$. This is because the framework may have the option to catch a sufficient example multiple times out of five.

\section{ISSUES}

There are likewise a few different issues to think about while assessing a biometrics framework's exhibition, like speed, client acknowledgement and so on You can, for instance, not utilize a biometrics framework in an ATM if it requires the framework a few minutes to check a client. Furthermore, if the clients don't believe the biometrics framework to be exact, they won't utilize it. Conversations over issues like these are normally gathered along with the FAR, FRR, CER and so forth into something many refer to as the Total System Performance (TSP).[2] Can separate the major boundaries in biometrics into four principal classifications: (I) exactness, (ii) scale, (iii) security, and (iv) protection. The basic guarantee of the ideal biometrics is that when a biometric identifier test is introduced to the biometric framework, it will offer the right choice. In contrast to the secret word or tokenbased framework, a viable biometric framework doesn't settle on wonderful match choices. It can make two fundamental kinds of blunders: I) false Match and ii) False positive match.

\section{False Match:}

In the bogus match kind of mistake, the biometric framework inaccurately proclaims an effective match between the info design and a non-coordinating with innovation in the data set (on account of distinguishing proof/screening) or the example related with a mistakenly asserted personality (on account of confirmation).

\section{Bogus Non-match:}

In the bogus non-coordinated with kind of blunder, the biometric framework inaccurately announces disappointment of match between the info design and a coordinating with innovation in the information base (recognizable proof/screening) or the example related with the accurately asserted character (check). It is more educational to report the framework exactness as far as a Receiver Operating Characteristic (ROC) bend. In any event, disregarding the necessities of complete mechanization and accepting the chance of good biometric signal obtaining from a good way, it is not difficult to note that there is a need to overcome any issues between the current innovation and execution prerequisites.

Acknowledge when contrasted with other example acknowledgement frameworks, the bogus dismissal of a client's case by a biometric framework is anything but a beneficial result since a manual distinguishing proof which is typically neither successful (for example, to check enlistment) nor achievable (e.g., enormous scope recognizable proof) must be done. Practical biometric frameworks also have huge disappointments in the inability to procure (FTA) and inability to select (FTE).

\section{EXPLANATIONS BEHIND IMPERFECT ACCURACY}

There are three essential purposes behind the flawed precision execution of a biometric framework. They are I) Information Limitation, ii) Representation Limitation, and iii) Invariance Limitation. [3]

\section{A. Data impediment:}

The invariant and unmistakable data content in the example tests might be innately restricted because of 
the inherent sign limit (e.g., individuality data limitation of the biometric identifier. For example, the data removed from the math is not exactly that of the fingerprints. Subsequently, hand math estimations can separate fewer personalities than the unique mark signal significantly under ideal conditions. Data impediment may likewise be expected to inadequately control biometric show by the clients or conflicting sign obtaining. The estimations of a biometric identifier obtained through different means limit the invariance across various examples of the example. For instance, data constraint happens when there is almost no crossover between the selected and test pictures in multiple stances and articulations. In such circumstances, even an ideal matcher neglects to offer a right coordinating with choice. An outrageous illustration of data limit is the point at which the individual doesn't have or can't present the precise biometric estimation required by the ID framework.

\section{B. Portrayal limit}

An ideal portrayal plot must be intended to hold all invariance and unfair data in the detected estimations. A common element extraction framework dependent on oversimplified models of biometric signal neglects to catch the extravagance of data in a sensible biometric sign, therefore bringing about the incorporation of mistaken components and rejection of natural elements. Thus, a critical part of real example space can't be taken care of by the biometric framework bringing about high FTA, FTE, FMR, and FNMR. For instance, the distinction data contained in the minutiabased portrayal of layouts represent commonplace "low quality" prints that can't be prepared by customary details based distinguishing proof frameworks, albeit the specialists regularly utilize such smirched patterns to settle on a solid match choice. Along these lines, regular portrayals and element extraction strategies are restricting the successful separation among the images.

\section{Invariance impediment}

At long last, in a portrayal conspire, the plan of an ideal matcher should completely display the invariance relationship in various examples from a similar class, in any event, when imaged under shifted show conditions. Once more, practically speaking (e.g., because of non-accessibility of an adequate number of preparing tests, uncontrolled or unforeseen fluctuation in the assortment conditions), a matcher may not effectively demonstrate the invariance relationship bringing about helpless matcher exactness.

\section{CONCLUSION}

The client validation, a fundamental piece of a DRM framework, decides if the client is approved to get to the substance. In a nonexclusive cryptographic framework, ownership of the unscrambling key is adequate proof to build up client validness. Cryptographic keys are long and irregular (e.g., 128 pieces for the high-level encryption standard (AES)), and they are hard to remember. Thus, the cryptographic keys are put away someplace (for instance, on a PC or a brilliant card) and delivered on the premise to any elective validation (e.g., secret phrase) system, that is, after guaranteeing that they are delivered to the approved clients. Most passwords are excessively basic such that they can be effortlessly speculated (particularly dependent on friendly designing strategies) or broken by direct word reference assaults. Can enhance large numbers of these constraints of the conventional passwords by consolidating better techniques for client validation.

Biometric validation is one such strategy that wipes out a large portion of the constraints different frameworks have. In Biometric confirmation, people are checked based on their physiological and social qualities like face, unique mark, hand calculation, iris, keystroke, signature, voice, and so on It is innately more dependable than secret key based confirmation because biometric attributes can't be lost or neglected (ex: passwords being lost or ignored); Biometric qualities are incredibly hard to duplicate, share, and appropriate (ex: passwords being declared in programmer sites) and require the individual at that point and place of verification (ex-scheming clients denying having shared the secret phrase). Biometric gives no degree for phoney since it requires additional time, cash, insight, and access advantages. It is far-fetched for a client to renounce an individual, the advanced substance utilizing biometrics. At last, biometrics is no simpler to break than another's; that is, all clients have a moderately equivalent security level, henceforth "simple to figure" biometrics, which can utilize to mount an assault against them, are generally missing. Hence, biometrics-based confirmation is likely to supplant secret key-based validation, either by building up the total verification instrument or by getting the conventional cryptographic keys containing the media record in a DRM framework. 
Numerous biometric attributes have been being used in different applications. Each biometric has its qualities and shortcomings, and the decision of the biometric relies upon the application. A solitary biometric cannot be anticipated to adequately meet every one of the necessities (e.g., precision, common sense, cost) of the relative multitude of uses (e.g., DRM, access control, government assistance dissemination). As such, no biometric is "ideal." The match between a particular biometric and a still up in the air is based on the necessities of the application and the properties of the biometric attributes.

\section{REFERENCES}

[1]. Whalberg M., "Biometric Security - Integration of Biometric Devices in Solaris”, University of Umea, 2000

[2]. Anil K. Jain, Sharath Pankanti, Salil Prabhakar, Lin Hong, and Arun Ross Michigan "Biometrics: A Grand Challenge”, State University, IBM T. J. Watson Research Center,

[3]. Nazeer Unnisa Nazima, Shahana Tanveer, Abdul Majeed, "Secure Public Key Protocol for Ad-Hoc Wireless Networks", International Journal of Computer Science and Network (IJCSN) Volume 1, Issue 6, Decembers 2012 www.ijcsn.org ISSN 2277-5420.

[4]. Soutar, Biometric System Security White Paper, Bioscrypt [Online]. Available: http://www.bioscrypt.com 